\title{
When some complement of a certain submodule is a summand
}

\author{
Yeliz KARA* \\ Bursa Uludă̆ University Faculty of Art and Science, Department of Mathematics, Bursa. \\ Geliş Tarihi (Received Date): 10.03.2021 \\ Kabul Tarihi (Accepted Date): 01.06.2021
}

\begin{abstract}
In this study, the modules whose p-submodules have a complement which is a direct summand are explored. The module theoretical properties such as direct sums and summands are investigated. As opposed to direct sums, this condition does not transfer to the direct summands. Thus, it is examined that under what conditions the direct summands fulfill the property. Examples are given to demonstrate the results.
\end{abstract}

Keywords: Complement submodule, extending module, projection invariant submodule.

\section{Belirli alt modüllerinin tümleyeni toplanan olan modüller}

$\ddot{\mathbf{O} z}$

Bu çalışmada p-alt modüllerinin tümleyeni dik toplanan olan modüller araştırılmıştır. Dik toplam ve dik toplanan gibi modül teorik özellikler incelenmiştir. Dik toplamın aksine, bu modül özelliği dik toplananlara taşınmadığından, hangi koşullar altında ilgili özelliğin dik toplananlara aktarıldiğı üzerinde çalışılmıştır. Elde edilen sonuçları niteleyen örneklere yer verilmiştir.

Anahtar kelimeler: Tümleyen alt modül, genişleyen modül, projeksiyon değişmez alt modül.

\section{Introduction}

All rings are associative with unity and modules are unital right modules. $\mathcal{R}$ and $\mathcal{M}$ denote a ring and a module, respectively. Recall that a module is extending [1], if every submodule is essential in a direct summand. Many authors have studied assorted

\footnotetext{
*Yeliz KARA, yelizkara@uludag.edu.tr, http://orcid.org/0000-0002-8001-6082
} 
generalizations of extending modules $[2,3,4,5,6]$. A submodule $\mathcal{V}$ of $\mathcal{M}$ is said to be projection invariant [7], if $\chi \mathcal{V} \subseteq \mathcal{V}, \forall \chi=\chi^{2} \in \operatorname{End}\left(\mathcal{M}_{\mathcal{R}}\right)$. A module is called $\pi$ extending [2], if every projection invariant submodule is essential in a direct summand. In this trend, a module $\mathcal{M}$ is called $C L S$ [5], if every $z$-closed submodule $\mathcal{L}$ (i.e., $\mathcal{M} / \mathcal{L}$ is nonsingular) of $\mathcal{M}$ is a direct summand. Motivated by the definition of $z$-closed submodules, a submodule $\mathcal{X}$ of $\mathcal{M}$ is called $p$-submodule [8], if $\mathcal{X}$ is projection invariant submodule of $\mathcal{M}$ such that $\mathcal{M} / \mathcal{X}$ is nonsingular.

The purpose of this study is to examine the class of $\pi^{p}$-module, i.e., whose $p$-submodule has a complement which is a direct summand. Extending and $\pi$-extending modules are contained in $\pi^{p}$-modules. In Section 2, fundamental properties and connections between the $\pi^{p}$ condition and related notions are obtained. In Section 3, we explore module theoretical properties encompassing direct sums and summands. The $\pi^{p}$-property is closed under direct sums. However, the forenamed condition does not transfer to the direct summands. Consequently, we explore under what conditions the module property transfers to the direct summands. We present examples to exhibit our results.

For the notation $\mathcal{Y} \leq \mathcal{M}, \mathcal{Y} \unlhd_{p} \mathcal{M}, \mathcal{Y} \leq_{e} \mathcal{M}, \mathcal{Y} \leq^{\oplus} \mathcal{M}, z_{2}(\mathcal{Y})$ and $\operatorname{End}\left(\mathcal{M}_{\mathcal{R}}\right)$, we mean that $\mathcal{Y}$ is a submodule of $\mathcal{M}, \mathcal{Y}$ is a projection invariant submodule of $\mathcal{M}, \mathcal{Y}$ is an essential submodule of $\mathcal{M}, \mathcal{Y}$ is a direct summand of $\mathcal{M}$, the second singular submodule of $\mathcal{M}$ and the endomorphism ring of $\mathcal{M}$, respectively. See [1, 6, 9], for unfamiliar notation.

\section{Preliminaries}

Fundamental properties and connections between the $\pi^{p}$ condition and related notions are obtained in this section. The first result is advantageous for the proof of our results.

Lemma 2.1 A module $\mathcal{M}$ is a $\pi^{p}$-module iff for each $p$-submodule $\mathcal{L}$ of $\mathcal{M}, \mathcal{L} \cap \mathcal{P}=0$ and $\mathcal{P} \oplus \mathcal{L} \leq_{e} \mathcal{M}$ for some $\mathcal{P} \leq^{\oplus} \mathcal{M}$.

Proof. Clear.

Proposition 2.2 Assume the following conditions:

(a) $\mathcal{M}$ is an extending module,

(b) $\mathcal{M}$ is a $C L S$-module,

(c) $\mathcal{M}$ is a $\pi$-extending module,

(d) $\mathcal{M}$ is a $\pi^{p}$-module.

Hence, $\quad(a) \Rightarrow(b) \Rightarrow(d)$ and $(a) \Rightarrow(c) \Rightarrow(d)$. However, the implications are irreversible.

Proof. These implications hold from definitions.

$(b) \nRightarrow(a)$. For any prime $q$, let $\mathcal{A}_{\mathbb{Z}}=(\mathbb{Z} / \mathbb{Z} q) \oplus\left(\mathbb{Z} / \mathbb{Z} q^{3}\right)$. By [5, Example 6], $\mathcal{A}$ holds $C L S$ property, but it does not fulfill the extending condition. 
(c) $\nRightarrow(a)$ Consider $\mathcal{T}=\left[\begin{array}{ll}\mathbb{Z} & \mathbb{Z} \\ 0 & \mathbb{Z}\end{array}\right]$ as a right $\mathcal{T}$-module. It follows from [2, Proposition 3.7] that $\mathcal{J}_{\mathcal{T}}$ is $\pi$-extending which it is not extending.

$(d) \nRightarrow(b)$. Let $\mathbb{Z}_{(2)}=\left\{\frac{s}{t}: s, t \in \mathbb{Z}, t\right.$ is odd $\}$ and $\mathcal{A}_{\mathbb{Z}}=\mathbb{Z} \oplus \mathbb{Z}_{(2)}$. Then, $\mathcal{A}$ does not hold $C L S$ property by [5, Example 15]. But, it is a $\pi^{p}$-module, as $\mathcal{A}$ is $\pi$-extending.

$(d) \nRightarrow(c)$. Assume $\mathcal{S}=\left[\begin{array}{cc}\mathcal{F} & \mathcal{V} \\ 0 & \mathcal{F}\end{array}\right]=\left\{\left[\begin{array}{cc}\kappa & \omega \\ 0 & \kappa\end{array}\right]: \kappa \in \mathcal{F}, \omega \in \mathcal{V}\right\}$, where $\mathcal{F}$ is a field and $\mathcal{V}_{\mathcal{F}}$ is a vector space with $\operatorname{dim}\left(\mathcal{V}_{\mathcal{F}}\right) \geq 2$. Note that $\mathcal{S}$ is an indecomposable $\mathcal{S}$-module. Hence, all submodules of $\mathcal{S}$ is projection invariant. Thus, $\mathcal{S}$ is the only $p$-submodule in itself. Hence, $\mathcal{S}_{\mathcal{S}}$ is a $\pi^{p}$-module. Nevertheless, $\mathcal{S}$ is not uniform, hence $\mathcal{S}_{\mathcal{S}}$ is not $\pi$-extending.

Proposition $2.3(i)$ Module properties of $\pi^{p}$ and $\pi$-extending agree for a nonsingular module.

(ii) Let $\mathcal{A}$ be a nonsingular indecomposable module. Then $\mathcal{A}$ is uniform $\Leftrightarrow \mathcal{A}$ is extending $\Leftrightarrow \mathcal{A}$ is $\pi$-extending $\Leftrightarrow \mathcal{A}$ is a $\pi^{p}$-module.

Proof. (i) Suppose $\mathcal{A}$ is a nonsingular $\pi^{p}$-module and $\mathcal{L} \unlhd_{p} \mathcal{A}$. Thus, there is a complement submodule $\mathcal{T}$ in $\mathcal{A}$ such that $\mathcal{L} \leq_{e} \mathcal{T}$. Since $\mathcal{A}$ is nonsingular, $\mathcal{T} \unlhd_{p} \mathcal{A}$ by [2, Lemma 2.3]. Consequently, $\mathcal{A} / \mathcal{T}$ is nonsingular by [6, Lemma 5.58 (ii)]. So, $\mathcal{T}$ is a $p$-submodule of $\mathcal{A}$. Then, $\mathcal{T} \cap \mathcal{P}=0$ and $\mathcal{T} \oplus \mathcal{P} \leq_{e} \mathcal{A}$ for some $\mathcal{P} \leq \oplus \mathcal{A}$, by Lemma 2.1. Then, $\mathcal{L} \cap \mathcal{P}=0$ and $\mathcal{L} \oplus \mathcal{P} \leq_{e} \mathcal{A}$. By [2, Corollary 3.2], $\mathcal{A}$ is $\pi$-extending. Proposition 2.2 yields the converse.

(ii) Part (i), Proposition 2.2 and [2, Proposition 3.8 (1)] complete the proof.

Consider $G_{\mathbb{Z}}=\mathbb{Z}^{\mathbb{N}}$ be the Specker group. Then, it is not $\pi$-extending by [4, Lemma 3.4]. Since $G_{\mathbb{Z}}$ is nonsingular, $G_{\mathbb{Z}}$ does not satisfy $\pi^{p}$ property by Proposition $2.3(i)$. However, the injective hull of $\mathrm{G}_{\mathbb{Z}}$ fulfills $\pi^{p}$ property. Therefore, the former module condition may not transfer to the submodules.

Proposition 2.4 Suppose $\mathcal{M}$ is a $\pi^{p}$-module and $\mathcal{L}$ is a $p$-submodule of $\mathcal{M}$. Thus, $\mathcal{L}$ holds $\pi^{p}$ property.

Proof. Suppose $\mathcal{L}$ is a $p$-submodule of $\mathcal{M}$ and $\mathcal{L}^{\prime}$ is a $p$-submodule of $\mathcal{L}$. By [8, Lemma 2.1], $\mathcal{L}^{\prime}$ is a $p$-submodule of $\mathcal{M}$. Thereby, $\mathcal{L}^{\prime} \cap \mathcal{P}=0$ and $\mathcal{L}^{\prime} \oplus \mathcal{P} \leq_{e} \mathcal{M}$, for some $\mathcal{P} \leq \oplus \mathcal{M}$. Accordingly $\mathcal{M}=\mathcal{P} \oplus \mathcal{P}^{\prime}$, for some $\mathcal{P}^{\prime} \leq \mathcal{M}$. Since $\mathcal{L} \unlhd_{p} M, \mathcal{L}=(\mathcal{L} \cap$ $\mathcal{P}) \oplus\left(\mathcal{L} \cap \mathcal{P}^{\prime}\right)$ by [7, p.50]. Observe that $\mathcal{L}^{\prime} \cap(\mathcal{L} \cap \mathcal{P})=0$ and $\mathcal{L} \cap\left(\mathcal{L}^{\prime} \oplus \mathcal{P}\right)=\mathcal{L}^{\prime} \oplus$ $(\mathcal{L} \cap \mathcal{P}) \leq_{e} \mathcal{L}$. Therefore, $\mathcal{L}$ is a $\pi^{p}$-module.

\section{Main results}

The module theoretical properties such as direct sums and summands are examined, and examples are given to demonstrate former results. 
Theorem 3.1 Assume $\mathcal{M}=\bigoplus_{\chi \in \Psi} \mathcal{M}_{\chi}$ such that $\mathcal{M}_{\chi}$ is a $\pi^{p}$-module for all $\chi \in \Psi$. Then $\mathcal{M}$ is a $\pi^{p}$-module.

Proof. Suppose $\mathcal{Y}$ is a $p$-submodule of $\mathcal{M}$ and $\emptyset \neq \Psi^{\prime} \subseteq \Psi$. Thus, the set

$\mathcal{B}=\left\{\left(\Psi^{\prime}, \mathcal{Y}, \mathcal{P}\right) \mid \Psi^{\prime} \subseteq \Psi, \quad \mathcal{Y} \cap \mathcal{P}=0\right.$ and $\quad \mathcal{Y} \oplus \mathcal{P} \leq_{e} \mathcal{M}$

where, $\quad \mathcal{Y}$ is a $p$-submodule of $\mathcal{M}$ and $\mathcal{P} \leq \oplus \mathcal{M}\}$

is partially ordered by

$$
\left(\Psi_{1}, \mathcal{Y}_{1}, \mathcal{P}_{1}\right) \leq\left(\Psi_{2}, \mathcal{Y}_{2}, \mathcal{P}_{2}\right) \Leftrightarrow \Psi_{1} \subseteq \Psi_{2}, \mathcal{Y}_{1} \leq \mathcal{Y}_{2}, \mathcal{P}_{1} \leq \mathcal{P}_{2}
$$

Notice that $\mathcal{B} \neq \varnothing$. Thus, there exists a maximal member $\left(\Psi_{1}, \mathcal{Y}_{1}, \mathcal{P}_{1}\right) \in \mathcal{B}$ by Zorn's Lemma. Assume $\Psi \neq \Psi_{1}$. Then, there is $\psi \in \Psi$ such that $\psi \notin \Psi_{1}$. Let $\Psi_{2}=\Psi_{1} \cup\{\psi\}$ and $\mathcal{M}^{\prime \prime}=\bigoplus_{\chi \in \Psi_{2}} \mathcal{M}_{\chi}=\bigoplus_{\chi \in \Psi_{1}} \mathcal{M}_{\chi} \oplus \mathcal{M}_{\psi}=\mathcal{M}_{1} \oplus \mathcal{M}_{\psi}$. Since $\mathcal{M}_{\chi}$ is a $\pi^{p}$-module, there is a $\mathcal{P}_{\psi} \leq{ }^{\oplus} \mathcal{M}_{\psi}$ such that $\mathcal{Y}_{\psi} \cap \mathcal{P}_{\psi}=0$ and $\mathcal{Y}_{\psi} \oplus \mathcal{P}_{\psi} \leq_{e} \mathcal{M}_{\psi}$ for $p$-submodule $\mathcal{Y}_{\psi}$ of $\mathcal{M}_{\psi}$. Note that $\mathcal{P}_{\psi} \cap \mathcal{P}_{1}=0$ and $\mathcal{P}^{\prime \prime}=\mathcal{P}_{\psi} \oplus \mathcal{P}_{1} \leq \oplus \mathcal{M}^{\prime \prime}$. For $p$-submodule $\mathcal{Y}_{1}$ of $\mathcal{M}_{1}$ and $\mathcal{Y}_{\psi}$ of $\mathcal{M}_{\psi}$, take $\mathcal{Y}_{1} \oplus \mathcal{Y}_{\psi} \leq \mathcal{M}^{\prime \prime}$. Observe that $\mathcal{Y}_{1} \oplus \mathcal{Y}_{\psi} \unlhd_{p} \mathcal{M}^{\prime \prime}$ and $\frac{\mathcal{M}^{\prime \prime}}{y_{1} \oplus \mathcal{Y}_{\psi}} \cong$ $\frac{\mathcal{M}_{1}}{y_{1}} \oplus \frac{\mathcal{M}_{\psi}}{y_{\psi}}$. It follows that $\frac{\mathcal{M}^{\prime \prime}}{y_{1} \oplus \mathcal{Y}_{\psi}}$ is nonsingular, so $\mathcal{Y}^{\prime \prime}=\mathcal{Y}_{1} \oplus \mathcal{Y}_{\psi}$ is a $p$-submodule of $\mathcal{M}^{\prime \prime}$. Thereby, $\mathcal{Y}^{\prime \prime} \cap \mathcal{P}^{\prime \prime}=0$ and $\mathcal{Y}^{\prime \prime} \oplus \mathcal{P}^{\prime \prime} \leq_{e} \mathcal{M}^{\prime \prime}$. Therefore, $\left(\Psi_{2}, \mathcal{Y}^{\prime \prime}, \mathcal{P}^{\prime \prime}\right) \in \mathcal{B}$. However, $\left(\Psi_{1}, \mathcal{Y}_{1}, \mathcal{P}_{1}\right) \leq\left(\Psi_{2}, \mathcal{Y}^{\prime \prime}, \mathcal{P}^{\prime \prime}\right)$ which contradicts to the maximality of $\left(\Psi_{1}, \mathcal{Y}_{1}, \mathcal{P}_{1}\right) \in \mathcal{B}$. Therefore, $\Psi=\Psi_{1}$, so $\mathcal{M}$ is a $\pi^{p}$-module.

As a consequence of above result, any direct sum of uniform (resp., extending, or $\pi$ extending) module and any free Abelian groups fulfill $\pi^{p}$ property. The next result provides that $\pi^{p}$ condition does not transfer to the direct summands, in general.

Example 3.2 Assume $T=\mathbb{R}\left[x_{1}, \ldots, x_{k}\right]$ with indeterminates $x_{1}, \ldots, x_{k}$ over $\mathbb{R}$, where $k$ is any odd integer with $k \geq 3$. Consider $\mathcal{R}=T / T t$ is a commutative Noetherian ring, where $t=x_{1}^{2}+\cdots+x_{k}^{2}-1$. Let $M=\mathcal{R}^{(k)}$. Thus, $M_{\mathcal{R}}$ is $\pi^{p}$-module by Theorem 3.1. Observe from [2, Example 5.5] that $M$ has a direct summand $\mathcal{P}_{\mathcal{R}}$ which is not $\pi$-extending. Nonsingularity of $\mathcal{R}$ yields that $\mathcal{P}_{\mathcal{R}}$ is not a $\pi^{p}$-module by Proposition 2.3(ii).

We deal with when the former module property transfers to the direct summands.

Proposition 3.3 Assume $\mathcal{V}_{1}$ and $\mathcal{V}_{2}$ are uniform modules such that $\mathcal{A}=\mathcal{V}_{1} \oplus \mathcal{V}_{2}$. Hence every direct summand of $\mathcal{A}$ holds $\pi^{p}$ property.

Proof. Suppose $0 \neq \mathcal{P} \leq \oplus \mathcal{A}$. By Theorem 3.1, $\mathcal{P}$ holds $\pi^{p}$ property when $\mathcal{P}=\mathcal{A}$. Assume $\mathcal{P} \neq \mathcal{A}$. Thus, $\mathcal{P}$ is uniform, so it satisfies $\pi^{p}$ property.

Proposition 3.4 If $\mathcal{A}_{\mathbb{Z}}=\bigoplus_{i \in I} \mathcal{V}_{i}$, where $\mathcal{V}_{i}(i \in I)$ is uniform, then every direct summand of $\mathcal{A}_{\mathbb{Z}}$ holds $\pi^{p}$ property.

Proof. Suppose $\mathcal{P} \leq \oplus \mathcal{A}$. By [4, Theorem 5.5], $\mathcal{P}=\bigoplus_{i \in I} \mathcal{U}_{i}$, where $\mathcal{U}_{i}$ is uniform. Hence Theorem 3.1 yields that $\mathcal{P}$ is a $\pi^{p}$-module. 
Note that a module fulfills SSP provided that the sum of two direct summands is a direct summand.

Proposition 3.5 $\mathcal{A}=\mathcal{A}_{1} \oplus \mathcal{A}_{2}$ for some $\mathcal{A}_{1}, \mathcal{A}_{2} \leq \mathcal{A}$ such that $\mathcal{A}_{2}$ is projection invariant. If $\mathcal{A}$ is a $\pi^{p}$-module with SSP, then $\mathcal{A}_{1}$ a $\pi^{p}$-module.

Proof. Let $\mathcal{L}$ be a $p$-submodule of $\mathcal{A}_{1}$. Thus, $\mathcal{L} \unlhd_{p} \mathcal{A}_{1}$ and $\mathcal{A}_{1} / \mathcal{L}$ is nonsingular. Observe from [2, Lemma 4.12] that $\mathcal{L} \oplus \mathcal{A}_{2} \unlhd_{p} \mathcal{A}$ and $\mathcal{A} /\left(\mathcal{L} \oplus \mathcal{A}_{2}\right) \cong \mathcal{A}_{1} / \mathcal{L}$ which give that $\mathcal{L} \oplus \mathcal{A}_{2}$ is a $p$-submodule of $\mathcal{A}$. Then there is a $\mathcal{P} \leq \oplus \mathcal{A}$ such that $\left(\mathcal{L} \oplus \mathcal{A}_{2}\right) \cap \mathcal{P}=0$ and $\left(\mathcal{L} \oplus \mathcal{A}_{2}\right) \oplus \mathcal{P} \leq_{e} \mathcal{A}$. Note that $\mathcal{A}_{1} \cap\left[\left(\mathcal{L} \oplus \mathcal{A}_{2}\right) \oplus \mathcal{P}\right]=\mathcal{L} \oplus\left[\mathcal{A}_{1} \cap\left(\mathcal{A}_{2} \oplus\right.\right.$ $\mathcal{P})] \leq_{e} \mathcal{A}_{1}$. Since $\left(\mathcal{L} \oplus \mathcal{A}_{2}\right) \cap \mathcal{P}=0, \mathcal{L} \cap\left[\mathcal{A}_{1} \cap\left(\mathcal{A}_{2} \oplus \mathcal{P}\right)\right]=0$. Moreover, $\mathcal{A}_{2} \cap$ $\mathcal{P}=0$ and $\mathcal{A}_{2} \oplus \mathcal{P}=\mathcal{A}_{2} \oplus\left[\mathcal{A}_{1} \cap\left(\mathcal{A}_{2} \oplus \mathcal{P}\right)\right]$ by modular law. Then, $\mathcal{A}_{2} \oplus$ $\mathcal{P} \leq \oplus \mathcal{A}$ by $S S P$ condition. It follows that $\mathcal{A}=\mathcal{A}_{2} \oplus\left[\mathcal{A}_{1} \cap\left(\mathcal{A}_{2} \oplus \mathcal{P}\right)\right] \oplus T$ for some $T \leq \mathcal{A}$. Hence, $\quad \mathcal{A}_{1}=\left[\mathcal{A}_{1} \cap\left(\mathcal{A}_{2} \oplus \mathcal{P}\right)\right] \oplus\left[\mathcal{A}_{1} \cap\left(\mathcal{A}_{2} \oplus T\right)\right]$ by modular law. Therefore, $\mathcal{A}_{1} \cap\left(\mathcal{A}_{2} \oplus \mathcal{P}\right) \leq \oplus \mathcal{A}_{1}$ which yields that $\mathcal{A}_{1}$ is a $\pi^{p}$-module.

A ring is said to be Abelian if every idempotent element is central.

Corollary 3.6 Suppose $\mathcal{A}$ is a $\pi^{p}$-module and $E n d\left(\mathcal{A}_{R}\right)$ is an Abelian ring with SSP. Then every direct summand of $\mathcal{A}_{R}$ fulfills $\pi^{p}$ condition.

Proof. Observe that $K \unlhd_{p} \mathcal{A}$ when $K \leq^{\oplus} \mathcal{A}$ and $\operatorname{End}\left(\mathcal{A}_{R}\right)$ is Abelian. It follows from [10, Corollary 2.4] that $\mathcal{A}$ has SSP. Thus, the proof is a consequent of Proposition 3.5.

Lemma 3.7 Let $\mathcal{A}=\mathcal{A}_{1} \oplus \mathcal{A}_{2}$ for some $\mathcal{A}_{1}, \mathcal{A}_{2} \leq \mathcal{A}$. Then $\mathcal{A}_{1}$ is a $\pi^{p}$-module iff for all $p$-submodule $\mathcal{Y}$ of $\mathcal{A}_{1}, \mathcal{A}_{2} \subseteq \mathcal{P}, \mathcal{P} \cap \mathcal{Y}=0$ and $\mathcal{P} \oplus \mathcal{Y} \leq_{e} \mathcal{A}$ for some $\mathcal{P} \leq \oplus \mathcal{A}$.

Proof. Let $\mathcal{A}_{1}$ provide $\pi^{p}$ condition and $\mathcal{Y}$ is a $p$-submodule of $\mathcal{A}_{1}$. Hence, $\mathcal{Y} \cap \mathcal{P}_{1}=0$ and $\mathcal{Y} \oplus \mathcal{P}_{1} \leq_{e} \mathcal{A}_{1}$ for some $\mathcal{P}_{1} \leq^{\oplus} \mathcal{A}_{1}$. It follows that $\mathcal{P}_{1} \oplus \mathcal{A}_{2} \leq \oplus \mathcal{A}$. Note that $\mathcal{A}_{2} \subseteq \mathcal{P}_{1} \oplus \mathcal{A}_{2},\left(\mathcal{P}_{1} \oplus \mathcal{A}_{2}\right) \cap \mathcal{Y}=0$ and $\mathcal{P}_{1} \oplus \mathcal{A}_{2} \oplus \mathcal{Y} \leq_{e} \mathcal{A}$. Conversely, suppose $\mathcal{A}_{1}$ fulfills the property. Take $\mathcal{L}$ as a $p$-submodule of $\mathcal{A}_{1}$. Then, $\mathcal{A}_{2} \subseteq \mathcal{P}^{\prime}, \mathcal{P}^{\prime} \cap \mathcal{L}=0$ and $\mathcal{P}^{\prime} \oplus \mathcal{L} \leq_{e} M$ for some $\mathcal{P}^{\prime} \leq^{\oplus} \mathcal{A}$, by hypothesis. Observe that $\mathcal{P}^{\prime} \cap \mathcal{A}_{1} \leq \oplus \mathcal{A}_{1}$. Thus, $\mathcal{L} \cap\left(\mathcal{P}^{\prime} \cap \mathcal{A}_{1}\right)=0$ and $\mathcal{L} \oplus\left(\mathcal{P}^{\prime} \cap \mathcal{A}_{1}\right)=\mathcal{A}_{1} \cap\left(\mathcal{L} \oplus \mathcal{P}^{\prime}\right) \leq_{e} \mathcal{A}_{1}$. Therefore, $\mathcal{A}_{1}$ is a $\pi^{p}$-module.

Theorem 3.8 Let $\mathcal{A}=\mathcal{A}_{1} \oplus \mathcal{A}_{2}$ is a $\pi^{p}$-module for some $\mathcal{A}_{1}, \mathcal{A}_{2} \leq \mathcal{A}$ such that $\mathcal{A}_{2}$ is projection invariant. Suppose $\mathcal{D} \oplus \mathcal{A}_{2} \leq{ }^{\oplus} \mathcal{A}$, for all $\mathcal{D} \leq \oplus \mathcal{A}$ with $\mathcal{D} \cap \mathcal{A}_{2}=0$.

Then $\mathcal{A}_{1}$ is a $\pi^{p}$-module.

Proof. Assume $\mathcal{L}$ is a $p$-submodule of $\mathcal{A}_{1}$. Note that $\mathcal{L} \oplus \mathcal{A}_{2} \unlhd_{p} \mathcal{A}$ by [2, Lemma 4.12]. Moreover, $\frac{\mathcal{A}}{\mathcal{L} \oplus \mathcal{A}_{2}} \cong \frac{\mathcal{A}_{1}}{\mathcal{L}}$ is nonsingular, so $\mathcal{L} \oplus \mathcal{A}_{2}$ is a $p$-submodule of $\mathcal{A}$. Consequently, $\mathcal{P} \cap\left(\mathcal{L} \oplus \mathcal{A}_{2}\right)=0, \quad \mathcal{P} \oplus \mathcal{L} \oplus \mathcal{A}_{2} \leq_{e} \mathcal{A}$ for some $\mathcal{P} \leq^{\oplus} \mathcal{A}$. Thus, Lemma 3.7 completes the proof.

Theorem 3.9 $\mathcal{A}$ is a $\pi^{p}$-module iff $\mathcal{A}=Z_{2}(\mathcal{A}) \oplus \mathcal{P}$ for some $\mathcal{P} \leq \mathcal{A}$, where $Z_{2}(\mathcal{A})$ and $\mathcal{P}$ hold $\pi^{p}$ condition.

Proof. Let $\mathcal{A}$ satisfy $\pi^{p}$ property and $\mathcal{W}=Z_{2}(\mathcal{A})$. Observe that $\mathcal{A} / \mathcal{W}$ is nonsingular, so $\mathcal{W}$ is $p$-submodule of $\mathcal{A}$. Hence, $\mathcal{W} \cap \mathcal{P}=0$ and $\mathcal{W} \oplus \mathcal{P} \leq_{e} \mathcal{A}$ for some $\mathcal{P} \leq^{\oplus} \mathcal{A}$. 
Thus, $\mathcal{W}=Z_{2}(\mathcal{A})=Z_{2}(\mathcal{P}) \oplus \mathcal{Z}_{2}\left(\mathcal{P}^{\prime}\right)$ for some $\mathcal{P}^{\prime} \leq \mathcal{A}$. As $\mathcal{W} \cap \mathcal{P}=0, Z_{2}(\mathcal{P})=0$. Then, $\mathcal{W}=\mathcal{Z}_{2}\left(\mathcal{P}^{\prime}\right) \leq \mathcal{P}^{\prime}$ which implies that $\mathcal{W} \leq_{e} \mathcal{P}^{\prime}$, as $\mathcal{W} \oplus \mathcal{P} \leq_{e} \mathcal{P}^{\prime} \oplus \mathcal{P}$. Therefore, $\mathcal{P}^{\prime} / \mathcal{W}$ is singular, so $\mathcal{W}=\mathcal{P}^{\prime}$ gives that $\mathcal{A}=\mathcal{W} \oplus \mathcal{P}$ for some $\mathcal{P} \leq \mathcal{A}$.

It is clear from Proposition 2.4 that $\mathcal{W}$ holds $\pi^{p}$ property. Consider the projection map $\pi: \mathcal{A} \rightarrow \mathcal{P}$. Then $\mathcal{W} \cap \mathcal{L}=0$ for some $p$-submodule $\mathcal{L}$ of $\mathcal{P}$. Observe that $\mathcal{W} \oplus \mathcal{L} \unlhd_{p} \mathcal{A}$ by [2, Lemma 4.12], and $\frac{\mathcal{A}}{\mathcal{W} \oplus \mathcal{L}} \cong \frac{\mathcal{P}}{\mathcal{L}}$ which implies that $\mathcal{W} \oplus \mathcal{L}$ is a $p$-submodule of $\mathcal{A}$. Hence, $\mathcal{D} \cap(\mathcal{W} \oplus \mathcal{L})=0, \mathcal{D} \oplus(\mathcal{W} \oplus \mathcal{L}) \leq_{e} \mathcal{A}$, for some $\mathcal{D} \leq \oplus \mathcal{A}$. Moreover, $\mathcal{W}=$ $Z_{2}(\mathcal{A})=Z_{2}(\mathcal{D}) \oplus Z_{2}\left(\mathcal{D}^{\prime}\right)$, where $\mathcal{A}=\mathcal{D} \oplus \mathcal{D}^{\prime}$ for some $\mathcal{D}^{\prime} \leq \mathcal{A}$. Hence $Z_{2}(\mathcal{D})=0$, so $\mathcal{W}=Z_{2}\left(\mathcal{D}^{\prime}\right) \leq \mathcal{D}^{\prime}$ and $\mathcal{D}^{\prime}=\mathcal{W} \oplus\left(\mathcal{D}^{\prime} \cap \mathcal{P}\right)$. Thus, $\mathcal{A}=\mathcal{D} \oplus \mathcal{D}^{\prime}=\mathcal{D} \oplus \mathcal{W} \oplus$ $\left(\mathcal{D}^{\prime} \cap \mathcal{P}\right)$, so $\mathcal{W} \oplus \mathcal{D} \leq^{\oplus} \mathcal{A}$. Clearly, $\mathcal{W} \oplus \mathcal{D}=\mathcal{W} \oplus \pi(\mathcal{D})$ for $\pi(\mathcal{D}) \leq \oplus \mathcal{P}$. Since $\mathcal{L} \oplus \pi(\mathcal{D}) \oplus \mathcal{W} \leq_{e} \mathcal{A}$, we have $\mathcal{L} \oplus \pi(\mathcal{D}) \leq_{e} \mathcal{P}$. Therefore, $\mathcal{P}$ is a $\pi^{p}$-module. Theorem 3.1 completes the converse.

We end up this section by the following example which exhibits Theorem 3.9 and the essential extensions of the $\pi^{p}$-modules.

Example 3.10 (i) Let $G=G_{1} \oplus G_{2}$, where $G_{1}$ and $G_{2}$ are Abelian groups with $G_{1}$ divisible and $G_{2}=\mathbb{Z} / \mathbb{Z} q^{s}$ such that $q$ is a prime, $s$ is a positive integer. Note $G_{1}=J \bigoplus$ $J^{\prime}$, where $J$ is torsionfree, $J^{\prime}$ is torsion. Thus, $G=J \oplus J^{\prime} \oplus G_{2}$ and $Z_{2}(G)=J^{\prime} \oplus G_{2}$. By Theorem 3.1, $G=Z_{2}(G) \oplus J$ provides $\pi^{p}$ condition.

(ii) Consider a principal ideal ring $\mathcal{B}$ which is not a complete discrete valuation ring. By [3, Theorem 19], there is a $\mathcal{B}$-module $\mathcal{A}$ such that $\mathcal{A}$ is indecomposable torsionfree with rank 2. Thus, $\mathcal{U}_{1} \oplus \mathcal{U}_{2} \leq_{e} \mathcal{A}$ for some uniform $\mathcal{U}_{1}, \mathcal{U}_{2} \leq \mathcal{A}$. By Theorem 3.1, $\mathcal{U}_{1} \oplus \mathcal{U}_{2}$ is a $\pi^{p}$-module. However, $\mathcal{A}_{\mathcal{B}}$ is not a $\pi^{p}$-module by Proposition 2.3 (ii).

\section{References}

[1] Dung, N. V., Huynh, D. V., Smith, P. F., Wisbauer, R., Extending Modules, Longman, Harlow, (1994).

[2] Birkenmeier, G. F., Tercan, A., Yücel, C. C., The extending condition relative to sets of submodules, Communications in Algebra 42, 764-778, (2014).

[3] Kaplansky, I., Infinite Abelian Groups, University of Michigan Press, (1969).

[4] Smith, P. F., Tercan A., Generalizations of CS-modules, Communications in Algebra 21(6), 1809-1847, (1993).

[5] Tercan, A., On CLS-modules, Rocky Mountain Journal of Mathematics, 25(4), 1557-1564, (1995).

[6] Tercan, A., Yücel, C. C., Module Theory, Extending Modules and Generalizations, Birkhäuser, Basel, (2016).

[7] Fuchs, L, Infinite Abelian Groups I, Academic Press, New York, (1970).

[8] Kara, Y., Modules whose $p$-submodules are direct summands, Turkish Journal of Mathematics, 42 (1), 28-33, (2018).

[9] Goodearl, K. R., Ring Theory: Nonsingular Rings and Modules, Dekker, New York, (1976).

[10] Garcia, J. L., Properties of direct summands of modules, Communications in Algebra, 17(1), 73-92, (1989). 